\title{
ON THE BASIS PROBLEM FOR SIEGEL-HILBERT MODULAR FORMS
}

\author{
JINGHUA KUANG
}

\begin{abstract}
In this paper, we mainly announce the result: every Siegel-Hilbert cuspform of weight divisible by $4 h$ and of square-free level relative to certain congruence subgroups is a linear combination of theta series.
\end{abstract}

\section{INTRODUCTION}

Theta series provides one of the two most explicit ways to construct holomorphic modular forms. The other way is by Eisenstein series. A virtue of theta series is that they are given in their Fourier expansions, with integral coefficients each of which is the number of lattice points of a certain length. One of the historic motivations to study modular forms was to study theta series. On the other hand, some modular forms, like the discriminant function $\Delta(z)$ of elliptic curve given by the lattice $\mathbb{Z}+\mathbb{Z} z$, are linear combinations of theta series. Indeed,

$$
\Delta(z)=\frac{691}{265344}(2 \pi)^{12}\left(\theta_{1}(z)-\theta_{2}(z)\right),
$$

where $\theta_{1}(z)$ and $\theta_{2}(z)$ are theta series attached to the quadratic lattices $\Gamma_{24}$ and $\Gamma_{8} \oplus \Gamma_{8} \oplus \Gamma_{8}$ respectively, see [Se]. Since the Fourier coefficients of $\Delta(z)$ are the famous Ramanujan partition numbers $\tau(n),(1)$ gives intrinsic relations between the partition numbers and the numbers of lattice points of certain lengths. This naturally leads to the so-called basis problem: Which modular forms are linear combinations of theta series and how to express them? In the light of Klingen's work on the decomposition of the space of modular forms as direct sums of subspaces of Eisenstein liftings of cuspforms (see [Kl] for the case of Siegel modular forms), the problem is reduced to a problem regarding cuspforms.

Received by the editors October 31, 1990 and, in revised form, February 11, 1991.

1980 Mathematics Subject Classification (1985 Revision). Primary 11F41, $11 \mathrm{~F} 27$. 
Solution of the basis problem has additional significance. Since the linear space of modular forms of a given level and a given weight decomposes into an orthogonal sum of a subspace of cuspforms and a subspace of Eisenstein series [Kl], the basis problem is actually a problem that asks whether theta series together with Eisenstein series are enough to generate all the modular forms (linearly). Particularly, if a cuspform is a linear combination of theta series with explicit coefficients $c_{1}, \ldots, c_{r}$, its Fourier coefficients are in the $\mathbb{Z}$-lattice generated by $c_{1}, \ldots, c_{r}$. To solve the basis problem is to determine the subspace generated by theta series. For such a subspace, it is interesting to study the integralitity of the Fourier coefficients and special values of L-functions attached to its elements. In the case of Siegel modular forms, if $\Phi$ is the Siegel operator $[\mathrm{Kl}]$, its kernel is the space of cuspforms. It is proved by Freitag [Fr] that $f$ has a preimage by $\Phi$ if and only if $f$ is a linear combination of theta series. Hence a solution to the basis problem regarding Siegel modular forms provides a determination of the image of $\Phi$, which is important in the study of Siegel modular forms.

The basis problem for elliptic modular forms has been studied in great detail by many mathematicians, among them are Hecke [He], Eichler [Ei], Waldspurger [Wa] and Hijikata, Pizer and Shemanske [HPS]. In [HPS], there are so far the most general results on the basis problem for elliptic modular forms. The basis problem for other modular forms has attracted attention recently. The problem for Hilbert modular form and for modular forms over $G L(2)$ can be solved by using the theory of 'newforms,' as it has been done for elliptic modular forms [Wa]. For Siegel modular forms, Ozeki [Oz], Weissauer [Wr], Böcherer [Bö] among others have achieved the same results in the case of level one. Böcherer has shown that every Siegel modular form of weight divisible by 4 is a linear combination of theta series with explicit coefficients, see [Bö]. T. Finnegan [Fi] generalized Böcherer's method and extended the results to the case of Siegel-Hilbert modular forms. (In contrast to Siegel modular forms for $\operatorname{Sp}(n, \mathbb{Q})$, a Siegel-Hilbert modular form is a modular form for $\operatorname{Sp}(n, F)$, where $F$ is a totally real number field.) $\mathrm{He}$ had to assume that the ground field has narrow class number 1 . However, there has been no result regarding the basis problem for Siegel modular forms of higher levels. Indeed, Böcherer pointed out in [Bö] that since there is not a proper extension of the theory of newforms in the case of Siegel 
modular forms, the problem for higher levels cannot be done in the same way as for elliptic modular forms of higher levels.

The main purpose of this paper is to announce a result which solves the basis problem for Siegel-Hilbert modular forms of square-free levels (relative to certain congruence subgroups).

\section{THE RESULTS}

Let $F$ be a totally real number field, $O_{F}$ be its ring of integers and $\mathfrak{n}$ a square-free ideal in $O_{F}$. Let $h$ be the narrow class number of $F$. Let $\Gamma_{0}(\mathfrak{n})$ be the congruence subgroup of $\operatorname{Sp}\left(n, O_{F}\right)$ defined as

$$
\Gamma_{0}(\mathfrak{n})=\left\{\left(\begin{array}{ll}
\alpha & \beta \\
\gamma & \delta
\end{array}\right) \in \operatorname{Sp}\left(n, O_{F}\right) \mid \gamma \equiv 0 \text { modulo } \mathfrak{n}\right\} .
$$

Let $\kappa$ be a positive integer greater than $(n+1) / 4 h$. Let $\rho$ be a Dirichlet character of conductor $\mathfrak{n}$. Denote by $C_{4 h \kappa}^{n}\left(\Gamma_{0}(\mathfrak{n}), \rho\right)$ the space of holomorphic Siegel-Hilbert modular cuspforms $f$ of weight $4 h \kappa$ of degree $n$, which transform under $\Gamma_{0}(\mathfrak{n})$ by

$$
f\left(\frac{\alpha z+\beta}{\gamma z+\delta}\right)=\rho(\operatorname{det} \alpha) \cdot \operatorname{det}(\gamma z+\delta)^{4 h \kappa} \cdot f(z)
$$

for $\left(\begin{array}{ll}\alpha & \beta \\ \gamma & \delta\end{array}\right) \in \Gamma_{0}(\mathfrak{n})$. Then our result is as follows:

Theorem 1. Every cuspform in $C_{4 h \kappa}^{n}\left(\Gamma_{0}(\mathfrak{n}), \rho\right)$ is a linear combination of theta series attached to quadratic forms in the genus of a certain totally positive-definite quadratic form.

In expressing such a cuspform as a linear combination of theta series, the linear coefficients are determined by a certain finite set of the Fourier coefficients of the cuspform in a way which is made explicit in the proof.

We also extend the results achieved by Finnegan [Fi] for SiegelHilbert modular forms of level one by removing the class-numberone condition on the ground field. Denote by $M_{4 h \kappa}^{n}\left(\operatorname{Sp}\left(n, O_{F}\right)\right)$ the space of holomorphic modular forms of level one of degree $n$. We have the following theorem.

Theorem 2. Every modular form in $M_{4 h \kappa}^{n}\left(\mathrm{Sp}\left(n, O_{F}\right)\right)$ is a linear combination of theta series attached to quadratic forms in a genus of a certain totally positive-definite quadratic form.

\section{SKETCH OF THE PROOF}

The two main ingredients in the proof are a decomposition formula and the Siegel-Weil formula [We2]. The idea to apply these 
two ingredients to study the basis problem is due to Böcherer. We proceed in an adelic way. By the strong approximation property of the symplectic groups $[\mathrm{Kn}]$, nothing is lost by doing so.

Let $m=8 h \kappa$. We construct a totally positive-definite symmetric $m \times m$ matrix $Q$ whose determinant is a square in $F^{\times}$ and which has 'level one.' Here by 'level one' we mean that for each finite place $\mathfrak{p}, x^{t} Q x \in 2 O_{\mathfrak{p}}^{*}$ for all $x \in M_{m 1}\left(O_{\mathfrak{p}}\right)$ and if $x \notin M_{m 1}\left(O_{\mathfrak{p}}\right)$, there exists $y \in M_{m 1}\left(O_{\mathfrak{p}}\right)$ such that $x^{t} Q y \notin O_{\mathfrak{p}}^{*}$, where $O_{\mathfrak{p}}$ is the valuation ring in $F_{\mathfrak{p}}$ and $O_{\mathfrak{p}}^{*}$ is the dual of $O_{\mathfrak{p}}$ relative to the standard additive character of $F_{\mathfrak{p}}$. We successfully obtain such a matrix by using the Hilbert class field extension of $F$ and a matrix related to the Lie algebra $E_{8}$. The divisibility of $m$ by $8 h$ is likely the necessary condition for the existence of such a matrix.

Let $\mathbb{A}_{F}$ be the adele ring of $F$. Given a Bruhat-Schwartz function $\varphi$ on $M_{m n}\left(\mathbb{A}_{F}\right)$, using the Weil representation $\mathbf{r}_{\mathbb{A}_{F}, Q}$ of $\operatorname{Sp}\left(n, \mathbb{A}_{F}\right)$ constructed with $Q$ [We1], we define an Eisenstein series

$$
E_{n, \varphi}(g)=\sum_{\gamma \in P_{n} \backslash \operatorname{Sp}\left(n, O_{F}\right)}\left(\mathbf{r}_{\mathbb{A}_{F}, Q}(\gamma g) \varphi\right)(0),
$$

where $P_{n}$ is the minimal parabolic subgroup of $\operatorname{Sp}\left(n, O_{F}\right)$. If the factor of $\varphi$ at an archimedean place is chosen to be

$$
\exp \left(-\pi \text { trace } x^{t} Q x\right),
$$

(2) is a holomorphic modular form on $\operatorname{Sp}\left(n, \mathbb{A}_{F}\right)$ of weight $m / 2$ when $m>2 n+2$.

We follow Garrett [Ga1] to decompose the Eisenstein series $E_{2 n, \varphi}$ restricted on the subgroup $\operatorname{Sp}\left(n, \mathbb{A}_{F}\right) \times \operatorname{Sp}\left(n, \mathbb{A}_{F}\right)$. It is reduced to evaluate an integral

$$
\int_{\operatorname{Sp}\left(r, F_{\mathfrak{p}}\right)} \eta_{\mathfrak{p}}^{(r)}\left(h_{\mathfrak{p}}\right) f^{\natural}\left(h h_{\mathfrak{p}}^{-1}\right) d h_{\mathfrak{p}}
$$

for each place $\mathfrak{p}$ of $F$ and each eigen-cuspform of the spherical Hecke algebras at good primes, where $\eta_{\mathfrak{p}}^{(r)}$ is a partial coefficient function of the $\mathfrak{p}$-component of the Weil representation $\mathbf{r}_{\mathrm{A}_{F}, Q}$ on the space generated by the Bruhat-Schwartz function $\varphi$, and $\nvdash$ is an involutional operator defined on the space of SiegelHilbert modular forms, $1 \leqslant r \leqslant n$. For the archimedean part, the computation is standard. For the good primes, we choose $\varphi$ 
to be the characteristic function of $M_{m n}\left(O_{p}\right)$ and apply the Satake map [Sa] to evaluate the integral (3). In a general decomposition of Eisenstein series, the bad-prime part plays a vital role. We achieve our explicit decomposition formula by constructing an adroit Schwartz-Bruhat function at bad primes. Such a function at a bad prime $\mathfrak{p}$ makes the partial coefficient function $\eta_{\mathfrak{p}}^{(r)}=0$ when $r<n$ and that $\eta_{\mathfrak{p}}^{(n)}$ has compact support

$$
\widetilde{\Gamma}_{0}^{\natural}(\mathfrak{p})=\left\{\left(\begin{array}{ll}
\alpha & \beta \\
\gamma & \delta
\end{array}\right) \in \operatorname{Sp}\left(n, O_{F}\right) \mid \beta \equiv 0 \text { modulo } \mathfrak{p}\right\} .
$$

Our Bruhat-Schwartz function can be decomposed as $\varphi=\varphi_{1} \otimes \varphi_{2}$. We obtain an explicit decomposition formula which we state as a theorem below.

Theorem 3.

$E_{2 n, \varphi}\left(g_{1}, g_{2}\right)=E_{n, \varphi_{1}}\left(g_{1}\right) \otimes E_{n, \varphi_{2}}\left(g_{2}\right)+\sum_{f} \frac{S(f)}{\langle f, f\rangle} \cdot f\left(g_{1}\right) \otimes f^{\natural}\left(g_{2}\right)$,

where the sum over $f$ runs over an orthogonal basis (with respect to the Petersson inner product $\langle\rangle$,$) of C_{m / 2}^{n}\left(\Gamma_{0}(\mathfrak{n}), \rho\right)$ consisting of simultaneous eigenfunctions of all spherical Hecke algebras at good primes, and $S(f)$ is the special value at $m / 2-n$ of the normalized symmetric-square L-function attached to $f$.

Now the Siegel-Weil formula tells us that any Eisenstein series of our type is an integral of theta series over the group

$$
O\left(Q, \mathbb{A}_{F}\right) / O(Q, F),
$$

where $O(Q$,$) is the orthogonal group of Q$. By a theorem due to Borel and Harish-Chandra [B-H], the totally positive definite condition of $Q$ implies that the integral is actually a finite sum. Combining the above and using a simple fact that a pullback (as above) of a theta series on a bigger group is a sum of products of theta series on smaller groups, we have that

$$
\sum_{f} \frac{S(f)}{\langle f, f\rangle} \cdot f\left(g_{1}\right) \otimes f^{\natural}\left(g_{2}\right)
$$

is a linear combination of products of theta series on variables $g_{1}, g_{2}$ attached to quadratic forms in the genus of $Q$. Then by an elementary linear algebra argument we obtain the proof of our Theorem 1.

Our proof for Theorem 2 is similar to that of Theorem 1. The decomposition formula that we have obtained and use to prove 
Theorem 2 appears in the same form as the one in [Fi], but is proved here under no conditions on the ground field $F$.

Full details will appear elsewhere $[\mathrm{Ku}]$.

\section{REMARKS}

1. We can apply our decomposition formulas and the Siegel-Weil formula to reprove a theorem originally due to Shimura [Sh] and generalized by Garrett [Ga2]: for the space $C_{4 h \kappa}^{n}\left(\Gamma_{0}(\mathfrak{n}), \rho\right)$, there exists an orthogonal basis with integral Fourier coefficients. Beyond this result, we have an assertion not found in [Sh] and [Ga2]: for a given cuspform $f$ with rational coefficients, there is effective bound for the denominators of its Fourier coefficients, arising from $S(f) /\langle f, f\rangle$ and from an invariant attached to the space.

2. A key point in successful treatment of the congruence subgroup $\Gamma_{0}(\mathfrak{n})$ is the construction of the Bruhat-Schwartz function such that the $\eta_{\mathfrak{p}}^{(n)}$ has compact support $\widetilde{\Gamma}_{0}^{\natural}(\mathfrak{p})$. Construction of such BruhatSchwartz functions for other types of congruence subgroups (with specified character) would yield the same results for those congruence subgroups.

3. Our method is readily applied to solve the same problem for modular forms on classical rational tube-type domains such as Hermitian modular forms and quaternion modular forms.

\section{ACKNOWLEDGMENT}

This paper is essentially an abstract of the author's Ph.D. thesis. The author would like to thank Professor Paul B. Garrett for his constant help and encouragement.

\section{REFERENCES}

[B-H] A. Borel and Harish-Chandra, Arithmetic subgroups of algebraic groups, Ann. of Math. (2) 75 (1962), 485-535.

[Bö] S. Böcherer, Siegel modular forms and theta series, Proc. Sympos. Pure Math., vol. 49, Amer. Math. Soc., Providence, RI, 1989.

[Ei] M. Eichler, The basis problem for modular forms and the traces of the Hecke operators, Lecture Notes in Math., vol. 320, Springer-Verlag, Berlin and New York, 1973.

[Fi] T. Finnegan, Siegel-Hilbert modular forms of level one over fields with narrow class number one are theta series, Ph.D. thesis, University of Minnesota, 1989. 
[Fr] E. Freitag, Stabile Modulformen, Math. Ann. 230 (1977), 162-170.

[Ga1] P. B. Garrett, Pullbacks of Eisenstein series, applications, Automorphic Forms of Several Variables, Taniguchi Symposium, Katata, 1983, Birkhäuser.

[Ga2] __ Arithmetic properties of Fourier-Jacobi expansions of automorphic forms in several variables, Amer. J. Math. 103 (1981), 1103-1134.

[He] E. Hecke, Analytische Arithmetik der Positiven Quadratischen Formen, Math. Werke, Vandenoek und Ruprecht Gottingen, 1959.

[HPS] H. Hijikata, A. Pizer, and T. Shemanske, The basis problem for modular forms on $\Gamma_{0}(N)$, Mem. Amer. Math. Soc., vol. 82, no. 418.

[Kl] H. Klingen, Introductory lectures on Siegel modular forms, Cambridge Univ. Press, 1990.

[Kn] M. Kneser, Strong approximation, Proc. Sympos. Math., vol. 9, Amer. Math. Soc., Providence, RI, 1966.

[Ku] J. Kuang, Certain Siegel-Hilbert modular forms of higher square-free levels are representable as theta series, Ph.D. Thesis, University of Minnesota, 1991.

[Oz] M. Ozeki, On basis problem for Siegel modular forms of degree 2, Acta Arith. 31 (1976), 17-30.

[Sa] I. Satake, Theory of spherical functions on reductive algebraic groups over p-adic fields, Inst. Hautes Ėtudes Sci. Publ. Math. 18 (1963), 1-69.

[Se] J. P. Serre, A course in arithmetic, Springer-Verlag, Berlin and New York, 1980.

[Sh] G. Shimura, On Fourier coefficients of modular forms of several variables, Nachr. Wiss. Gott. 17 (1975), 261-268.

[Wa] J. Waldspurger, Engendrement par des séries theta de certain espaces de formes modulaires, Invent. Math. 50 (1979), 135-168.

[We1] A. Weil, Sur certaines groupes d'operateurs unitaires, Acta Math. 113 (1965), $1-87$.

[We2] __ Sur la formule de Siegel dans la théorie des groupes classiques, Acta Math. 113 (1965), 1-87.

[Wr] R. Weissauer, Stabile Modulformen und Eisenteineihen, Lecture Notes in Math., vol. 1219, Springer-Verlag, Berlin and New York, 1986.

School of Mathematics, University of Minnesota, Twin Cities, MinNESOTA 55455.

E-mail address: jkuang@ux.acs.edu 
